Article

\title{
A Third Order Newton-Like Method and Its Applications
}

\author{
D. R. Sahu ${ }^{1, *}$, Ravi P. Agarwal ${ }^{2}$ and Vipin Kumar Singh ${ }^{3}$
}

1 Department of Mathematics, Banaras Hindu University, Varanasi-221005, India

2 Department of Mathematics, Texas A\&M University-Kingsville, Kingsville, TX 78363-8202, USA;

Ravi.Agarwal@tamuk.edu

3 Department of Mathematics, Banaras Hindu University, Varanasi-221005, India;

vipinkumarsingh666@gmail.com

* Correspondence: drsahudr@gmail.com

Received: 8 October 2018; Accepted: 7 December 2018; Published: 30 December 2018

\begin{abstract}
In this paper, we design a new third order Newton-like method and establish its convergence theory for finding the approximate solutions of nonlinear operator equations in the setting of Banach spaces. First, we discuss the convergence analysis of our third order Newton-like method under the $\omega$-continuity condition. Then we apply our approach to solve nonlinear fixed point problems and Fredholm integral equations, where the first derivative of an involved operator does not necessarily satisfy the Hölder and Lipschitz continuity conditions. Several numerical examples are given, which compare the applicability of our convergence theory with the ones in the literature.
\end{abstract}

Keywords: nonlinear operator equation; Fréchet derivative; $\omega$-continuity condition; Newton-like method; Frédholm integral equation

\section{Introduction}

Our purpose of this paper is to compute solution of nonlinear operator equation of the form

$$
F(x)=0
$$

where $F: D \subset X \rightarrow Y$ is a nonlinear operator defined on an open convex subset $D$ of a Banach space $X$ with values into a Banach space $Y$.

A lot of challenging problems in physics, numerical analysis, engineering, and applied mathematics are formulated in terms of finding roots of the equation of the form Equation (1). In order to solve such problems, we often use iterative methods. There are many iterative methods available in literature. One of the central method for solving such problems is the Newton method [1,2] defined by

$$
x_{n+1}=x_{n}-\left(F_{x_{n}}^{\prime}\right)^{-1} F\left(x_{n}\right)
$$

for each $n \geq 0$, where $F_{x}^{\prime}$ denotes the Fréchet derivative of $F$ at point $x \in D$.

The Newton method and the Newton-like method are attractive because it converges rapidly from any sufficient initial guess. A number of researchers [3-20] have generalized and established local as well as semilocal convergence analysis of the Newton method Equation (2) under the following conditions:

(a) Lipschitz condition: $\left\|F_{x}^{\prime}-F_{y}^{\prime}\right\| \leq K\|x-y\|$ for all $x, y \in D$ and for some $K>0$;

(b) Hölder Lipschitz condition: $\left\|F_{x}^{\prime}-F_{y}^{\prime}\right\| \leq K\|x-y\|^{p}$ for all $x, y \in D$ and for some $p \in(0,1]$ and $K>0$; 
(c) $\omega$-continuity condition: $\left\|F_{x}^{\prime}-F_{y}^{\prime}\right\| \leq \omega(\|x-y\|)$ for all $x, y \in D$, where $\omega:[0, \infty) \rightarrow[0, \infty)$ is a nondecreasing and continuous function.

One can observe that the condition (c) is more general than the conditions (a) and (b). One can find numerical examples where the Lipschitz condition (a) and the Hölder continuity condition (b) on the first Fréchet derivative do not hold, but the $\omega$-continuity condition (c) on first Fréchet derivative holds (see Example 1, [21]).

On the other hand, many mathematical problems such as differential equations, integral equations, economics theory, game theory, variational inequalities, and optimization theory $([22,23])$ can be formulated into the fixed point problem:

$$
\text { Find } x \in C \text { such that } x=G(x) \text {, }
$$

where $G: C \rightarrow X$ is an operator defined on a nonempty subset $C$ of a metric space $X$. The easiest iterative method for constructing a sequence is Picard iterative method [24] which is given by

$$
x_{n+1}=G\left(x_{n}\right)
$$

for each $n \geq 0$. The Banach contraction principle (see $[1,22,23,25]$ ) provides sufficient conditions for the convergence of the iterative method Equation (4) to the fixed point of $G$. Banach spaces have more geometrical stricture with respect to metric spaces. For study fixed points of nonlinear smooth operators, Banach space structure is required. More details of Banach space theory and fixed point theory of nonlinear operators can be found in $[1,22,23,26-28]$.

The Newton method and its variant $[29,30]$ are also used to solve the fixed point problem of the form:

$$
(I-G)(x)=0,
$$

where $I$ is the identity operator defined on $X$ and $G: D \subset X \rightarrow X$ is a nonlinear Fréchet differentiable operator defined on an open convex subset $D$ of a Banach space $X$. For finding approximate solution of the Equation (5), Bartle [31] used the Newton-like iterative method of the form

$$
x_{n+1}=x_{n}-\left(I-G_{y_{n}}^{\prime}\right)^{-1}\left(I-G\left(x_{n}\right)\right)
$$

for each $n \geq 0$, where $G_{x}^{\prime}$ is Fréchet derivative of $G$ at point $x \in D$ and $\left\{y_{n}\right\}$ is the sequence of arbitrary points in $D$ which are sufficiently closed to the desired solution of the Equation (5). Bartle [31] has discussed the convergence analysis of the form Equation (6) under the assumption that $G$ is Fréchet differentiable at least at desired points and a modulus of continuity is known for $G^{\prime}$ as a function of $x$. The Newton method Equation (2) and the modified Newton method are the special cases of the form Equation (6).

Following the idea of Bartle [31], Rall [32] introduced the following Stirling method for finding a solution of the fixed point problem Equation (5):

$$
\left\{\begin{array}{l}
y_{n}=G\left(x_{n}\right) \\
x_{n+1}=x_{n}-\left(I-G_{y_{n}}^{\prime}\right)^{-1}\left(x_{n}-G\left(x_{n}\right)\right)
\end{array}\right.
$$

for each $n \geq 0$. Many researchers [33-35] have studied the Stirling method Equation (7) and established local as well as semilocal convergence analysis of the Stirling-like method.

Recently, Parhi and Gupta [21,36] have discussed the semilocal convergence analysis of the following Stirling-like iterative method for computing a solution of operator Equation (5):

$$
\left\{\begin{array}{l}
z_{n}=G\left(x_{n}\right) \\
y_{n}=x_{n}-\left(I-G_{z_{n}}^{\prime}\right)^{-1}\left(x_{n}-G\left(x_{n}\right)\right) \\
x_{n+1}=y_{n}-\left(I-G_{z_{n}}^{\prime}\right)^{-1}\left(y_{n}-G\left(y_{n}\right)\right)
\end{array}\right.
$$


for each $n \geq 0$. More precisely, Parhi and Gupta [21] have studied the semilocal convergence analysis of Equation (8) for computing a solution of the operator Equation (5), where $G: D \subset X \rightarrow X$ is a nonlinear Fréchet differentiable operator defined on an open convex subset $D$ under the condition:

$$
(\Omega)\left\|G_{x}^{\prime}\right\| \leq k \text { for all } x \in D \text { and for some } k \in\left(0, \frac{1}{3}\right]
$$

There are some nonlinear Fréchet differentiable operators $G: D \subset X \rightarrow X$ defined on an open convex subset $D$ which fail to satisfy the condition $(\Omega)$ (see Example 1). Therefore, ref. [21] (Theorem 1) is not applicable for such operators. So, there is the following natural question:

Problem 1. Is it possible to develop the Stirling-like iterative method for computing a solution of the operator Equation (5), where the condition $(\Omega)$ does not hold?

The main purpose of this paper is to design a new Newton-like method for solving the operator Equation (1) and provide an affirmative answer of the Problem 1. We prove our proposed Newton-like method has $R$-order of convergence at least $2 p+1$ under the $\omega$-continuity condition and it covers a wide variety of iterative methods. We derive the Stirling-like iterative method for computing a solution of the fixed point problem Equation (5), where $(\Omega)$ does not hold and hence it gives an affirmative answer to Question 1 and generalizes the results of Parhi and Gupta [21,36] in the context of the condition $(\Omega)$.

In Section 2, we summarize some known concepts and results. In Section 3, we introduce a new Newton-like method for solving the operator Equation (1) and establish convergence theory of the proposed Newton-like method. In Section 4, we derive the Stirling-like iterative method from the proposed Newton-like method and establish a convergence theorem for computing a solution of the fixed point problem. Applications to Fredholm integral equations are also presented in Section 5, together with several numerical examples, which compare the applicability of our iterative technique with the ones in the literature.

\section{Preliminary}

In this section, we discuss some technical results. Throughout the paper, we denote $B(X, Y)$ a collection of bounded linear operators from a Banach space $X$ into a Banach space $Y$ and $B(X)=B(X, X)$. For some $r>0, B_{r}[x]$ and $B_{r}(x)$ are the closed and open balls with center $x$ and radius $r$, respectively, $\mathbb{N}_{0}=\mathbb{N} \cup\{0\}$ and $\Phi$ denote the collection of nonnegative, nondecreasing, continuous real valued functions defined on $[0, \infty)$.

Lemma 1. (Rall [37] (p. 50)) Let $\mathcal{L}$ be a bounded linear operator on a Banach space X. Then $\mathcal{L}^{-1}$ exists if and only if there is a bounded linear operator $M$ in X such that $M^{-1}$ exists and

$$
\|M-\mathcal{L}\|<\frac{1}{\left\|M^{-1}\right\|}
$$

If $\mathcal{L}^{-1}$ exists, then we have

$$
\left\|\mathcal{L}^{-1}\right\| \leq \frac{\left\|M^{-1}\right\|}{1-\left\|1-M^{-1} \mathcal{L}\right\|} \leq \frac{\left\|M^{-1}\right\|}{1-\left\|M^{-1}\right\|\|M-\mathcal{L}\|}
$$

Lemma 2. Let $0<k \leq \frac{1}{3}$ be a real number. Assume that $q=\frac{1}{p+1}+k^{p}$ for any $p \in(0,1]$ and the scalar equation

$$
\left(1-k^{p}(1+q t)^{p} t\right)^{p+1}-\left(\frac{q^{p} t^{p}}{p+1}+k^{p}\right)^{p} q^{p} t^{2 p}=0
$$

has a minimum positive root $\alpha$. Then we have the following: 
(1) $q>k$ for all $p \in(0,1]$.

(2) $\alpha \in(0,1)$.

Proof. (1) This part is obvious. Indeed, we have

$$
\frac{1}{p+1}+k^{p}-\frac{1}{3}=\frac{2-p}{3(1+p)}+k^{p}>0
$$

for all $p \in(0,1]$ and $0<k \leq \frac{1}{3}$.

(2) Set

$$
g(t)=\left(1-k^{p}(1+q t)^{p} t\right)^{p+1}-\left(\frac{q^{p} t^{p}}{p+1}+k^{p}\right)^{p} q^{p} t^{2 p}
$$

It is clear from the definition of $g(t)$ that $g(0)>0, g(1)<0$ and $g^{\prime}(t)<0$ in $(0,1)$. Therefore, $g(t)$ is decreasing in $(0,1)$ and hence the Equation (9) has a minimum positive $\operatorname{root} \alpha \in(0,1)$. This completes the proof.

Lemma 3. Let $b_{0} \in(0, \alpha)$ be a number such that $k^{p}\left(1+q b_{0}\right)^{p} b_{0}<1$, where $k, p, \alpha$ and $q$ are same as in Lemma 2. Define the real sequences $\left\{b_{n}\right\},\left\{\theta_{n}\right\}$ and $\left\{\gamma_{n}\right\}$ by

$$
\begin{gathered}
b_{n+1}=\frac{\left(\frac{q^{p} b_{n}^{p}}{p+1}+k^{p}\right)^{p} q^{p} b_{n}^{2 p}}{\left(1-k^{p}\left(1+q b_{n}\right)^{p} b_{n}\right)^{p+1}} b_{n} \\
\theta_{n}=\frac{\left(\frac{q^{p} b_{n}^{p}}{p+1}+k^{p}\right) q b_{n}^{2}}{1-k^{p}\left(1+q b_{n}\right)^{p} b_{n}}, \quad \gamma_{n}=\frac{1}{1-k^{p}\left(1+q b_{n}\right)^{p} b_{n}}
\end{gathered}
$$

for each $n \in \mathbb{N}_{0}$. Then we have the following:
(1) $\frac{\left(\frac{q^{p} b_{0}^{p}}{p+1}+k^{p}\right)^{p} q^{p} b_{0}^{2 p}}{\left(1-k^{p}\left(1+q b_{0}\right)^{p} b_{0}\right)^{p+1}}<1$.
(2) The sequence $\left\{b_{n}\right\}$ is decreasing, that is $b_{n+1} \leq b_{n}$ for all $n \in \mathbb{N}_{0}$.
(3) $k^{p}\left(1+q b_{n}\right)^{p} b_{n}<1$ for all $n \in \mathbb{N}_{0}$.
(4) $b_{n+1} \leq \xi^{(2 p+1)^{n}} b_{n}$ for all $n \in \mathbb{N}_{0}$.
(5) $\theta_{n} \leq \xi^{\frac{(2 p+1)^{n}-1}{p}} \theta$ for all $n \in \mathbb{N}_{0}$, where $\theta_{0}=\theta$ and $\xi=\gamma_{0} \theta^{p}$.

Proof. (1) Since the scalar equation $g(t)=0$ defined by Equation (9) has a minimum positive root $\alpha \in(0,1)$ and $g(t)$ is decreasing in $(0,1)$ with $g(0)>0$ and $g(1)<0$. Therefore, $g(t)>0$ in the interval $(0, \alpha)$ and hence

$$
\frac{\left(\frac{q^{p} b_{0}^{p}}{p+1}+k^{p}\right)^{p} q^{p} b_{0}^{2 p}}{\left(1-k^{p}\left(1+q b_{0}\right)^{p} b_{0}\right)^{p+1}}<1 .
$$

(2) From (1) and Equation (10), we have $b_{1} \leq b_{0}$. This shows that (2) is true for $n=0$. Let $j \geq 0$ be a fixed positive integer. Assume that (2) is true for $n=0,1,2, \cdots, j$. Now, using Equation (10), we have

$$
b_{j+2}=\frac{\left(\frac{q^{p} b_{j+1}^{p}}{p+1}+k^{p}\right)^{p} q^{p} b_{j+1}^{2 p}}{\left(1-k^{p}\left(1+q b_{j+1}\right)^{p} b_{j+1}\right)^{p+1}} b_{j+1} \leq \frac{\left(\frac{q^{p} b_{j}^{p}}{p+1}+k^{p}\right)^{p} q^{p} b_{j}^{2 p}}{\left(1-k^{p}\left(1+q b_{j}\right)^{p} b_{n}\right)^{p+1}} b_{j}=b_{j+1} .
$$

Thus (2) holds for $n=j+1$. Therefore, by induction, (2) holds for all $n \in \mathbb{N}_{0}$. 
(3) Since $b_{n}<b_{n-1}$ for each $n=1,2,3, \cdots$ and $k^{p}\left(1+q b_{0}\right)^{p} b_{0}<1$ for all $p \in(0,1]$, it follows that

$$
k^{p}\left(1+q b_{n}\right)^{p} b_{n}<k^{p}\left(1+q b_{0}\right)^{p} b_{0}<1
$$

(4) From (3), one can easily prove that the sequences $\left\{\gamma_{n}\right\}$ and $\left\{\theta_{n}\right\}$ are well defined. Using Equations (10) and (11), one can easily observe that

$$
b_{n+1}=\gamma_{n} \theta_{n}^{p} b_{n}
$$

for each $n \in \mathbb{N}_{0}$. Put $n=0$ and $n=1$ in Equation (12), we have

$$
b_{1}=\gamma_{0} \theta^{p} b_{0}=\xi^{(2 p+1)^{0}} b_{0}
$$

and

$$
\begin{aligned}
b_{2} & =\frac{\left(\frac{q^{p} b_{1}^{p}}{p+1}+k^{p}\right)^{p} q^{p} b_{1}^{2 p}}{\left(1-k^{p}\left(1+q b_{1}\right)^{p} b_{1}\right)^{p+1}} b_{1} \\
& \leq \frac{\left(\frac{q^{p} b_{0}^{p}}{p+1}+k^{p}\right)^{p} q^{p}\left(\xi b_{0}\right)^{2 p}}{\left(1-k^{p}\left(1+q b_{0}\right)^{p} b_{0}\right)^{p+1}} b_{1} \\
\leq & \xi^{2 p} \frac{\left(\frac{q^{p} b_{0}^{p}}{p+1}+k^{p}\right)^{p} q^{p} b_{0}^{2 p}}{\left(1-k^{p}\left(1+q b_{0}\right)^{p} b_{0}\right)^{p+1}} b_{1} \\
& =\xi^{2 p} \gamma_{0} \theta^{p} b_{1}=\xi^{2 p+1} b_{1} .
\end{aligned}
$$

Hence (4) holds for $n=0$ and $n=1$. Let $j>1$ be a fixed integer. Assume that (4) holds for each $n=0,1,2 \cdots, j$. From Equations (11) and (12), we have

$$
\begin{aligned}
b_{j+2} & =\frac{\left(\frac{q^{p} b_{j+1}^{p}}{p+1}+k^{p}\right)^{p} q^{p} b_{j+1}^{2 p}}{\left(1-k^{p}\left(1+q b_{j+1}\right)^{p} b_{j+1}\right)^{p+1}} b_{j+1} \\
& \leq \frac{\left(\frac{q^{p} b_{j+1}^{p}}{p+1}+k^{p}\right)^{p} q^{p}\left(\xi^{(2 p+1)^{j}} b_{j}\right)^{2 p}}{\left(1-k^{p}\left(1+q b_{j+1}\right)^{p} b_{j+1}\right)^{p+1}} b_{j+1} \\
& \leq\left(\xi^{2 p(2 p+1)^{j}}\right) \frac{\left(\frac{q^{p} b_{j}^{p}}{p+1}+k^{p}\right)^{p} q^{p} b_{j}^{2 p}}{\left(1-k^{p}\left(1+q b_{j}\right)^{p} b_{j}\right)^{p+1}} b_{j+1} \\
& \leq \xi^{2 p(2 p+1)^{j}} \xi^{2 p(2 p+1)^{j-1}} \cdots \xi^{2 p(2 p+1)} \xi^{(2 p+1)} b_{j+1} \\
& =\xi^{(2 p+1)^{j+1}} b_{j+1} \cdot
\end{aligned}
$$

Thus (4) holds for $n=j+1$. Therefore, by induction, (4) holds for all $n \in \mathbb{N}_{0}$.

(5) From Equation (11) and (4), one can easily observe that

$$
\theta_{1}=\frac{\left(\frac{q^{p} b_{1}^{p}}{p+1}+k^{p}\right) q b_{1}^{2}}{1-k^{p}\left(1+q b_{1}\right)^{p} b_{1}} \leq \frac{\left(\frac{q^{p} b_{0}^{p}}{p+1}+k^{p}\right) q\left(\xi b_{0}\right)^{2}}{1-k^{p}\left(1+q b_{0}\right)^{p} b_{0}} \leq \xi^{\frac{(2 p+1)^{1}-1}{p}} \theta .
$$


Hence (5) holds for $n=1$. Let $j>1$ be a fixed integer. Assume that (5) holds for each $n=0,1,2 \cdots, j$. From Equation (11), we have

$$
\begin{aligned}
\theta_{j+1} & =\frac{\left(\frac{q^{p} b_{j+1}^{p}}{p+1}+k^{p}\right) q b_{j+1}^{2}}{1-k^{p}\left(1+q b_{j+1}\right)^{p} b_{j+1}} \\
& \leq \frac{\left(\frac{q^{p} b_{0}^{p}}{p+1}+k^{p}\right) q\left(\xi^{\frac{(2 p+1)^{j+1}-1}{2 p}} b_{0}\right)^{2}}{1-k^{p}\left(1+q b_{0}\right)^{p} b_{0}} \\
& =\xi^{\frac{(2 p+1)^{j+1}-1}{p}} \theta .
\end{aligned}
$$

Thus (5) holds for $n=j+1$. Therefore, by induction, $(v)$ holds for all $n \in \mathbb{N}_{0}$. This completes the proof.

\section{Computation of a Solution of the Operator Equation (1)}

Let $X$ and $Y$ be Banach spaces and $D$ be a nonempty open convex subset of $X$. Let $F: D \subset X \rightarrow Y$ be a nonlinear operator such that $F$ is Fréchet differentiable at each point of $D$ and let $L \in B(Y, X)$ such that $(I-L F)(D) \subseteq D$. To solve the operator Equation (1), we introduce the Newton-like algorithm as follows:

Starting with $x_{0} \in D$ and after $x_{n} \in D$ is defined, we define the next iterate $x_{n+1}$ as follows:

$$
\left\{\begin{array}{l}
z_{n}=(I-L F)\left(x_{n}\right), \\
y_{n}=\left(I-F_{z_{n}}^{\prime-1} F\right)\left(x_{n}\right), \\
x_{n+1}=\left(I-F_{z_{n}}^{\prime-1} F\right)\left(y_{n}\right)
\end{array}\right.
$$

for each $n \in \mathbb{N}_{0}$.

If we take $X=Y, F=I-G$ and $L=I \in B(X)$ in Equation (13), then the iteration process Equation (13) reduces to the Stirling-like iteration process Equation (8).

Before proving the main result of the paper, we establish the following:

Proposition 1. Let $D$ be a nonempty open convex subset of a Banach space $X, F: D \subset X \rightarrow Y$ be a Fréchet differentiable at each point of $D$ with values in a Banach space $Y$ and $L \in B(Y, X)$ such that $(I-L F)(D) \subseteq D$. Let $\omega:[0, \infty) \rightarrow[0, \infty)$ be a nondecreasing and continuous real-valued function. Assume that $F$ satisfies the following conditions:

(1) $\left\|F_{x}^{\prime}-F_{y}^{\prime}\right\| \leq \omega(\|x-y\|)$ for all $x, y \in D$;

(2) $\left\|I-L F_{x}^{\prime}\right\| \leq c$ for all $x \in D$ and for some $c \in(0, \infty)$.

Define a mapping $T: D \rightarrow D$ by

$$
T(x)=(I-L F)(x)
$$

for all $x \in D$. Then we have

$$
\left\|I-F_{T x}^{\prime-1} F_{T y}^{\prime}\right\| \leq\left\|F_{T x}^{\prime-1}\right\| \omega(c\|x-y\|)
$$

for all $x, y \in D$.

Proof. For any $x, y \in D$, we have 


$$
\begin{aligned}
\left\|I-F_{T x}^{\prime-1} F_{T y}^{\prime}\right\| & \leq\left\|F_{T x}^{\prime-1}\right\|\left\|F_{T x}^{\prime}-F_{T y}^{\prime}\right\| \\
& \leq\left\|F_{T x}^{\prime-1}\right\| \omega(\|T x-T y\|) \\
& =\left\|F_{T x}^{\prime-1}\right\| \omega(\|x-y-L(F(x)-F(y))\|) \\
& =\left\|F_{T x}^{\prime-1}\right\| \omega\left(\left\|x-y-L \int_{0}^{1} F_{y+t(x-y)}^{\prime}(x-y) d t\right\|\right) \\
& \leq\left\|F_{T x}^{\prime-1}\right\| \omega\left(\int_{0}^{1}\left\|I-L F_{y+t(x-y)}^{\prime}\right\| d t\|x-y\|\right) \\
& \leq\left\|F_{T x}^{\prime-1}\right\| \omega(c\|x-y\|) .
\end{aligned}
$$

This completes the proof.

Now, we are ready to prove our main results for solving the problem Equation (1) in the framework of Banach spaces.

Theorem 1. Let $D$ be a nonempty open convex subset of a Banach space $X, F: D \subset X \rightarrow Y$ a Fréchet differentiable at each point of $D$ with values in a Banach space $Y$ and $L \in B(Y, X)$ such that $(I-L F)(D) \subseteq D$. Let $x_{0} \in D$ be such that $z_{0}=x_{0}-L F\left(x_{0}\right)$ and $F_{z_{0}}^{\prime-1} \in B(Y, X)$ exist. Let $\omega \in \Phi$ and let $\alpha$ be the solution of the Equation (9). Assume that the following conditions hold:

(C1) $\left\|F_{x}^{\prime}-F_{y}^{\prime}\right\| \leq \omega(\|x-y\|)$ for all $x, y \in D$;

(C2) $\left\|I-L F_{x}^{\prime}\right\| \leq k$ for all $x \in D$ and for some $k \in\left(0, \frac{1}{3}\right]$;

(C3) $\left\|F_{z_{0}}^{\prime-1}\right\| \leq \beta$ for some $\beta>0$;

(C4) $\left\|F_{z_{0}}^{\prime-1} F\left(x_{0}\right)\right\| \leq \eta$ for some $\eta>0$;

(C5) $\omega(t s) \leq t^{p} \omega(s), s \in[0, \infty), t \in[0,1]$ and $p \in(0,1]$;

(C6) $b_{0}=\beta \omega(\eta)<\alpha, q=\frac{1}{p+1}+k^{p}, \theta=\frac{\left(\frac{q^{p} b_{0}^{p}}{p+1}+k^{p}\right) q b_{0}^{2}}{1-k^{p}\left(1+q b_{0}\right)^{p} b_{0}}$ and $B_{r}\left[x_{0}\right] \subset D$, where $r=\frac{1+q}{1-\theta} \eta$.

Then we have the following:

(1) The sequence $\left\{x_{n}\right\}$ generated by Equation (13) is well defined, remains in $B_{r}\left[x_{0}\right]$ and satisfies the following estimates:

$$
\left\{\begin{array}{l}
\left\|y_{n-1}-z_{n-1}\right\| \leq k\left\|y_{n-1}-x_{n-1}\right\| \\
\left\|x_{n}-y_{n-1}\right\| \leq q b_{n-1}\left\|y_{n-1}-x_{n-1}\right\| \\
\left\|x_{n}-x_{n-1}\right\| \leq\left(1+q b_{n-1}\right)\left\|y_{n-1}-x_{n-1}\right\| \\
F_{z_{n}}^{\prime-1} \text { exists and }\left\|F_{z_{n}}^{-1}\right\| \leq \gamma_{n-1}\left\|F_{z_{n-1}}^{\prime-1}\right\| \\
\left\|y_{n}-x_{n}\right\| \leq \theta_{n-1}\left\|y_{n-1}-x_{n-1}\right\| \leq \theta^{n}\left\|y_{0}-x_{0}\right\| \\
\left\|F_{z_{n}}^{\prime-1}\right\| \omega\left(\left\|y_{n}-x_{n}\right\|\right) \leq b_{n}
\end{array}\right.
$$

for all $n \in \mathbb{N}$, where $z_{n}, y_{n} \in B_{r}\left[x_{0}\right]$, the sequences $\left\{b_{n}\right\},\left\{\theta_{n}\right\}$, and $\left\{\gamma_{n}\right\}$ are defined by Equations (10) and (11), respectively.

(2) The sequence $\left\{x_{n}\right\}$ converges to the solution $x^{*} \in B_{r}\left[x_{0}\right]$ of the Equation (1).

(3) The priory error bounds on $x^{*}$ is given by:

$$
\left\|x_{n}-x^{*}\right\| \leq \frac{\left(1+q b_{0}\right) \eta}{\xi^{1 / 2 p^{2}}\left(1-\xi^{\frac{(2 p+1)^{n}}{p}} \gamma_{0}^{-\frac{1}{p}}\right) \gamma_{0}^{n / p}}\left(\xi^{1 / 2 p^{2}}\right)^{(2 p+1)^{n}}
$$

for each $n \in \mathbb{N}_{0}$.

(4) The sequence $\left\{x_{n}\right\}$ has $R$-order of convergence at least $2 p+1$. 
Proof. (1) First, we show that Equation (15) is true for $n=1$. Since $x_{0} \in D, y_{0}=x_{0}-F_{z_{0}}^{\prime-1} F\left(x_{0}\right)$ is well defined. Note that

$$
\left\|y_{0}-x_{0}\right\|=\left\|F_{z_{0}}^{\prime-1} F\left(x_{0}\right)\right\| \leq \eta<r .
$$

Hence $y_{0} \in B_{r}\left[x_{0}\right]$. Using Equation (13), we have

$$
\begin{aligned}
\left\|y_{0}-z_{0}\right\| & =\left\|-F_{z_{0}}^{\prime-1} F\left(x_{0}\right)+L F\left(x_{0}\right)\right\| \\
& =\left\|y_{0}-x_{0}-L F_{z_{0}}^{\prime}\left(y_{0}-x_{0}\right)\right\| \\
& \leq\left\|I-L F_{z_{0}}^{\prime}\right\|\left\|y_{0}-x_{0}\right\| \\
& \leq k\left\|y_{0}-x_{0}\right\| .
\end{aligned}
$$

By Proposition 1 and (C2), we have

$$
\begin{aligned}
\left\|x_{1}-y_{0}\right\| & =\left\|F_{z_{0}}^{\prime-1}\left(F\left(y_{0}\right)-F\left(x_{0}\right)-F_{z_{0}}^{\prime}\left(y_{0}-x_{0}\right)\right)\right\| \\
& \leq \int_{0}^{1}\left\|F_{z_{0}}^{\prime-1}\left(F_{x_{0}+t\left(y_{0}-x_{0}\right)}^{\prime}-F_{y_{0}}^{\prime}+F_{y_{0}}^{\prime}-F_{z_{0}}^{\prime}\right)\right\|\left\|y_{0}-x_{0}\right\| d t \\
& \leq \beta\left[\int_{0}^{1}\left\|\left(F_{x_{0}+t\left(y_{0}-x_{0}\right)}^{\prime}-F_{y_{0}}^{\prime}\right)\right\| d t+\left\|F_{y_{0}}^{\prime}-F_{z_{0}}^{\prime}\right\|\right]\left\|y_{0}-x_{0}\right\| \\
& =\beta\left[\int_{0}^{1} \omega\left((1-t)\left\|y_{0}-x_{0}\right\|\right) d t+\omega\left(\left\|y_{0}-z_{0}\right\|\right)\right]\left\|y_{0}-x_{0}\right\| \\
& =\beta\left[\int_{0}^{1}(1-t)^{p} \omega\left(\left\|y_{0}-x_{0}\right\|\right) d t+\omega\left(k\left\|y_{0}-x_{0}\right\|\right)\right]\left\|y_{0}-x_{0}\right\| \\
& =\beta\left[\int_{0}^{1}(1-t)^{p} \omega\left(\left\|y_{0}-x_{0}\right\|\right) d t+k^{p} \omega\left(\left\|y_{0}-x_{0}\right\|\right)\right]\left\|y_{0}-x_{0}\right\| \\
& \leq \beta\left[\frac{1}{p+1}+k^{p}\right] \omega\left(\left\|y_{0}-x_{0}\right\|\right)\left\|y_{0}-x_{0}\right\| \\
& \leq q \beta \omega(\eta)\left\|y_{0}-x_{0}\right\| \leq q b_{0}\left\|y_{0}-x_{0}\right\| .
\end{aligned}
$$

Thus we have

$$
\begin{aligned}
\left\|x_{1}-x_{0}\right\| & \leq\left\|x_{1}-y_{0}\right\|+\left\|y_{0}-x_{0}\right\| \leq q b_{0}\left\|y_{0}-x_{0}\right\|+\left\|y_{0}-x_{0}\right\| \\
& \leq\left(1+q b_{0}\right)\left\|y_{0}-x_{0}\right\|<r
\end{aligned}
$$

which shows that $x_{1} \in B_{r}\left[x_{0}\right]$. Note that $z_{1}=(I-L F)\left(x_{1}\right) \in D$. Using Proposition 1 and $(C 3)-(C 5)$, we have

$$
\begin{aligned}
\left\|I-F_{z_{0}}^{\prime-1} F_{z_{1}}^{\prime}\right\| & \leq\left\|F_{z_{0}}^{\prime-1}\right\| \omega\left(k\left\|x_{1}-x_{0}\right\|\right) \\
& \leq \beta \omega\left(k\left(1+q b_{0}\right)\left\|y_{0}-x_{0}\right\|\right) \\
& \leq \beta k^{p}\left(1+q b_{0}\right)^{p} \omega\left(\left\|y_{0}-x_{0}\right\|\right) \\
& \leq k^{p}\left(1+q b_{0}\right)^{p} \beta \omega(\eta) \\
& \leq\left(k\left(1+q b_{0}\right)\right)^{p} b_{0}<1 .
\end{aligned}
$$

Therefore, by Lemma $1, F_{z_{1}}^{\prime-1}$ exists and

$$
\left\|F_{z_{1}}^{\prime-1}\right\| \leq \frac{\left\|F_{z_{0}}^{\prime-1}\right\|}{1-\left(k\left(1+q b_{0}\right)\right)^{p} b_{0}}=\gamma_{0}\left\|F_{z_{0}}^{\prime-1}\right\|
$$

Subsequently, we have 


$$
\begin{aligned}
& \left\|y_{1}-x_{1}\right\|=\left\|F_{z_{1}}^{\prime-1} F\left(x_{1}\right)\right\| \\
& =\left\|F_{z_{1}}^{\prime-1}\left(F\left(x_{1}\right)-F\left(y_{0}\right)-F_{z_{0}}^{\prime}\left(x_{1}-y_{0}\right)\right)\right\| \\
& \leq\left\|F_{z_{1}}^{\prime-1}\right\|\left[\int_{0}^{1}\left\|\left(F_{y_{0}+t\left(x_{1}-y_{0}\right)}^{\prime}-F_{y_{0}}^{\prime}\right)\right\| d t+\left\|F_{y_{0}}^{\prime}-F_{z_{0}}^{\prime}\right\|\right]\left\|x_{1}-y_{0}\right\| \\
& \leq\left\|F_{z_{1}}^{\prime-1}\right\|\left[\frac{1}{p+1} \omega\left(\left\|x_{1}-y_{0}\right\|\right)+\omega\left(k\left\|y_{0}-x_{0}\right\|\right)\right]\left\|x_{1}-y_{0}\right\| \\
& \leq\left\|F_{z_{1}}^{\prime-1}\right\|\left[\frac{1}{p+1} \omega\left(q b_{0}\left\|x_{0}-y_{0}\right\|\right)+k^{p} \omega\left(\left\|y_{0}-x_{0}\right\|\right)\right] q b_{0}\left\|x_{0}-y_{0}\right\| \\
& \leq\left\|F_{z_{1}}^{\prime-1}\right\|\left[\frac{q^{p} b_{0}^{p}}{p+1} \omega\left(\left\|y_{0}-x_{0}\right\|\right)+k^{p} \omega\left(\left\|y_{0}-x_{0}\right\|\right)\right] q b_{0}\left\|y_{0}-x_{0}\right\| \\
& \leq \gamma_{0}\left[\frac{q^{p} b_{0}^{p}}{p+1}+k^{p}\right] \beta \omega(\eta) q b_{0}\left\|y_{0}-x_{0}\right\| \\
& \leq \frac{\left(\frac{q^{p} b_{0}^{p}}{p+1}+k^{p}\right) q b_{0}^{2}}{1-\left(k\left(1+q b_{0}\right)\right)^{p} b_{0}}\left\|y_{0}-x_{0}\right\| \\
& \leq \theta\left\|y_{0}-x_{0}\right\| \text {. }
\end{aligned}
$$

From Equations (16) and (18), we have

$$
\begin{aligned}
\left\|y_{1}-x_{0}\right\| & \leq\left\|y_{1}-x_{1}\right\|+\left\|x_{1}-x_{0}\right\| \\
& \leq \theta\left\|y_{0}-x_{0}\right\|+\left(1+q b_{0}\right)\left\|y_{0}-x_{0}\right\| \\
& \leq\left(1+q b_{0}\right) \theta\left\|y_{0}-x_{0}\right\|+\left(1+q b_{0}\right)\left\|y_{0}-x_{0}\right\| \\
& \leq\left(1+q b_{0}\right)(1+\theta) \eta<r
\end{aligned}
$$

and

$$
\begin{aligned}
\| z_{1}-x_{0} \mid & \leq\left\|z_{1}-y_{1}\right\|+\left\|y_{1}-x_{1}\right\|+\left\|x_{1}-x_{0}\right\| \\
& \leq(1+k)\left\|y_{1}-x_{1}\right\|+\left(1+q b_{0}\right)\left\|y_{0}-x_{0}\right\| \\
& \leq(1+q) \theta \eta+(1+q) \eta \\
& =(1+q)(1+\theta) \eta<r .
\end{aligned}
$$

This shows that $z_{1}, y_{1} \in B_{r}\left[x_{0}\right]$. From Equations (17) and (18), we get

$$
\begin{aligned}
\left\|F_{z_{1}}^{\prime-1}\right\| \omega\left(\left\|y_{1}-x_{1}\right\|\right) & \leq \gamma_{0}\left\|F_{z_{0}}^{\prime-1}\right\| \omega\left(\theta\left\|y_{0}-x_{0}\right\|\right) \\
& \leq \gamma_{0} \theta^{p} \beta \omega(\eta) \\
& \leq \gamma_{0} \theta^{p} b_{0}=b_{1} .
\end{aligned}
$$

Thus we see that Equation (15) holds for $n=1$.

Let $j>1$ be a fixed integer. Assume that Equation (15) is true for $n=1,2, \cdots, j$. Since $x_{j} \in B_{r}\left[x_{0}\right]$, it follows $z_{j}=(I-L F)\left(x_{j}\right) \in D$. Using (C3), (C4), Equations (13) and (15), we have

$$
\begin{aligned}
\left\|y_{j}-z_{j}\right\| & =\left\|L F\left(x_{j}\right)-F_{z_{j}}^{\prime-1} F\left(x_{j}\right)\right\|=\left\|\left(L-F_{z_{j}}^{\prime-1}\right) F\left(x_{j}\right)\right\| \\
& =\left\|\left(L-F_{z_{j}}^{\prime-1}\right) F_{z_{j}}^{\prime}\left(x_{j}-y_{j}\right)\right\| \\
& \leq\left\|I-L F_{z_{j}}^{\prime}\right\|\left\|y_{j}-x_{j}\right\| \\
& \leq k\left\|y_{j}-x_{j}\right\| .
\end{aligned}
$$

Using Equations (13) and (19), we have 


$$
\begin{aligned}
\left\|x_{j+1}-y_{j}\right\| & =\left\|F_{z_{j}}^{\prime-1} F\left(y_{j}\right)\right\| \\
& \leq\left\|F_{z_{j}}^{\prime-1}\right\|\left\|F\left(y_{j}\right)-F\left(x_{j}\right)-F_{z_{j}}^{\prime}\left(y_{j}-x_{j}\right)\right\| \\
& \leq\left\|F_{z_{j}}^{\prime-1}\right\|\left[\int_{0}^{1}\left\|F_{x_{j}+t\left(y_{j}-x_{j}\right)}^{\prime}-F_{z_{j}}^{\prime}\right\| d t\right]\left\|y_{j}-x_{j}\right\| \\
& \leq\left\|F_{z_{j}}^{\prime-1}\right\|\left[\int_{0}^{1}\left\|F_{x_{j}+t\left(y_{j}-x_{j}\right)}^{\prime}-F_{y_{j}}^{\prime}\right\| d t+\left\|F_{y_{j}}^{\prime}-F_{z_{j}}^{\prime}\right\|\right]\left\|y_{j}-x_{j}\right\| \\
& =\left\|F_{z_{j}}^{\prime-1}\right\|\left[\int_{0}^{1} \omega\left(x_{j}+t\left(y_{j}-x_{j}\right)-y_{j}\right) d t+\omega\left(\left\|y_{j}-z_{j}\right\|\right)\right]\left\|y_{j}-x_{j}\right\| \\
& \leq\left\|F_{z_{j}}^{\prime-1}\right\|\left[\int_{0}^{1} \omega\left((1-t)\left\|y_{j}-x_{j}\right\|\right) d t+\omega\left(k\left\|y_{j}-x_{j}\right\|\right)\right]\left\|y_{j}-x_{j}\right\| \\
& \leq\left\|F_{z_{j}}^{\prime-1}\right\|\left[\int_{0}^{1}\left((1-t)^{p}+k^{p}\right) \omega\left(\left\|y_{j}-x_{j}\right\|\right) d t\right]\left\|y_{j}-x_{j}\right\| \\
& \leq\left\|F_{z_{j}}^{\prime-1}\right\|\left[\frac{1}{p+1}+k^{p}\right] \omega\left(\left\|y_{j}-x_{j}\right\|\right)\left\|y_{j}-x_{j}\right\| \\
& =q\left\|F_{z_{j}}^{\prime-1}\right\| \omega\left(\left\|y_{j}-x_{j}\right\|\right)\left\|y_{j}-x_{j}\right\| \\
& =q b_{j}\left\|y_{j}-x_{j}\right\| .
\end{aligned}
$$

From Equation (20), we have

$$
\begin{aligned}
\left\|x_{j+1}-x_{j}\right\| & \leq\left\|x_{j+1}-y_{j}\right\|+\left\|y_{j}-x_{j}\right\| \\
& \leq q b_{j}\left\|y_{j}-x_{j}\right\|+\left\|y_{j}-x_{j}\right\| \\
& \leq\left(1+q b_{j}\right)\left\|y_{j}-x_{j}\right\| .
\end{aligned}
$$

Using Equations (20) and (21) and the triangular inequality, we have

$$
\begin{aligned}
\left\|x_{j+1}-x_{0}\right\| & \leq \sum_{s=0}^{j}\left\|x_{s+1}-x_{s}\right\| \\
& \leq \sum_{s=0}^{j}\left(1+q b_{s}\right)\left\|y_{s}-x_{s}\right\| \\
& \leq \sum_{s=0}^{j}\left(1+q b_{0}\right) \theta^{s}\left\|y_{0}-x_{0}\right\| \\
& \leq\left(1+q b_{0}\right) \frac{1-\theta^{j+1}}{1-\theta} \eta \\
& \leq \frac{(1+q) \eta}{1-\theta}=r,
\end{aligned}
$$

which implies that $x_{k+1} \in B_{r}\left[x_{0}\right]$. Again, by using Proposition 1, (C2), (C5), and Equation (21), we have

$$
\begin{aligned}
\left\|I-F_{z_{j}}^{\prime-1} F_{z_{j+1}}^{\prime}\right\| & \leq\left\|F_{z_{j}}^{\prime-1}\right\| \omega\left(k\left\|x_{j+1}-x_{j}\right\|\right) \\
& \leq\left\|F_{z_{j}}^{\prime-1}\right\| k^{p}\left(1+q b_{j}\right)^{p} \omega\left(\left\|y_{j}-x_{j}\right\|\right) \\
& \leq k^{p}\left(1+q b_{j}\right)^{p} b_{j}<1 .
\end{aligned}
$$

Therefore, by Lemma $1, F_{z_{j+1}}^{\prime-1}$ exists and

$$
\left\|F_{z_{j+1}}^{\prime-1}\right\| \leq \frac{\left\|F_{z_{j}}^{\prime-1}\right\|}{1-k^{p}\left(1+q b_{j}\right)^{p} b_{j}}=\gamma_{j}\left\|F_{z_{j}}^{\prime-1}\right\| .
$$

Using Equations (13), (C2), and (21), we have 


$$
\begin{aligned}
& \left\|y_{j+1}-x_{j+1}\right\|=\left\|F_{z_{j+1}}^{\prime-1} F\left(x_{j+1}\right)\right\| \\
& =\left\|F_{z_{j+1}}^{\prime-1}\left(F\left(x_{j+1}\right)-F\left(y_{j}\right)-F_{z_{j}}^{\prime}\left(x_{j+1}-y_{j}\right)\right)\right\| \\
& \leq\left\|F_{z_{j+1}}^{\prime-1}\right\|\left[\int_{0}^{1}\left\|F_{y_{j}+t\left(x_{j+1}-y_{j}\right)}^{\prime}-F_{y_{j}}^{\prime}\right\| d t+\left\|F_{y_{j}}^{\prime}-F_{z_{j}}^{\prime}\right\|\right]\left\|x_{j+1}-y_{j}\right\| \\
& \leq\left\|F_{z_{j+1}}^{\prime-1}\right\|\left[\int_{0}^{1} \omega\left(t\left\|x_{j+1}-y_{j}\right\|\right) d t+\omega\left(\left\|y_{j}-z_{j}\right\|\right)\right]\left\|x_{j+1}-y_{j}\right\| \\
& \leq\left\|F_{z_{j+1}}^{\prime-1}\right\|\left[\int_{0}^{1} \omega\left(t q b_{j}\left\|y_{j}-x_{j}\right\|\right) d t+\omega\left(k\left\|y_{j}-x_{j}\right\|\right)\right] q b_{j}\left\|y_{j}-x_{j}\right\| \\
& \leq \gamma_{j}\left\|F_{z_{j}}^{\prime-1}\right\|\left[\frac{q^{p} b_{j}^{p}}{p+1} \omega\left(\left\|y_{j}-x_{j}\right\|\right)+k^{p} \omega\left(\left\|y_{j}-x_{j}\right\|\right)\right] q b_{j}\left\|y_{j}-x_{j}\right\| \\
& =\gamma_{j}\left[\frac{q^{p} b_{j}^{p}}{p+1}+k^{p}\right]\left\|F_{z_{j}}^{\prime-1}\right\| \omega\left(\left\|y_{j}-x_{j}\right\|\right) q b_{j}\left\|y_{j}-x_{j}\right\| \\
& \leq \gamma_{j}\left[\frac{q^{p} b_{j}^{p}}{p+1}+k^{p}\right] q b_{j}^{2}\left\|y_{j}-x_{j}\right\| \\
& \leq \theta_{j}\left\|y_{j}-x_{j}\right\| \leq \theta^{j+1}\left\|y_{0}-x_{0}\right\|, \\
& \left\|y_{j+1}-x_{0}\right\| \leq\left\|y_{j+1}-x_{j+1}\right\|+\left\|x_{j+1}-x_{0}\right\| \\
& \leq \theta^{j+1}\left\|y_{0}-x_{0}\right\|+\sum_{s=0}^{j}\left\|x_{s+1}-x_{s}\right\| \\
& \leq \theta^{j+1}\left\|y_{0}-x_{0}\right\|+\sum_{s=0}^{j}\left(1+q b_{0}\right) \theta^{s}\left\|y_{0}-x_{0}\right\| \\
& \leq\left(1+q b_{0}\right) \sum_{s=0}^{j+1} \theta^{s} \eta \\
& \leq \frac{(1+q) \eta}{1-\theta}=r
\end{aligned}
$$

and

$$
\begin{aligned}
\left\|z_{j+1}-x_{0}\right\| & \leq\left\|z_{j+1}-y_{j+1}\right\|+\left\|y_{j+1}-x_{j+1}\right\|+\left\|x_{j+1}-x_{0}\right\| \\
& \leq(1+k)\left\|y_{j+1}-x_{j+1}\right\|+\sum_{s=0}^{j}\left(1+q b_{0}\right) \theta^{s} \eta \\
& \leq(1+q) \theta^{j+1} \eta+\sum_{s=0}^{j}(1+q) \theta^{s} \eta \\
& \leq \sum_{s=0}^{j+1}(1+q) \theta^{s} \eta<r
\end{aligned}
$$

which implies that $z_{j+1}, y_{j+1} \in B_{r}\left(x_{0}\right)$. Also, we have

$$
\begin{aligned}
\left\|F_{z_{j+1}}^{\prime-1}\right\| \omega\left(\left\|y_{j+1}-x_{j+1}\right\|\right) & \leq \gamma_{j}\left\|F_{z_{j}}^{\prime-1}\right\| \omega\left(\theta_{j}\left\|y_{j}-x_{j}\right\|\right) \\
& \leq \gamma_{j} \theta_{j}^{p}\left\|F_{z_{j}}^{\prime-1}\right\| \omega\left(\left\|y_{j}-x_{j}\right\|\right) \\
& \leq \gamma_{j} \theta_{j}^{p} b_{j}=b_{j+1} .
\end{aligned}
$$

Hence we conclude that Equation (15) is true for $n=j+1$. Therefore, by induction, Equation (15) is true for all $n \in \mathbb{N}_{0}$. 
(2) First, we show that the sequence $\left\{x_{n}\right\}$ is a Cauchy sequence. For this, letting $m, n \in \mathbb{N}_{0}$ and using Lemma 3, we have

$$
\begin{aligned}
\left\|x_{m+n}-x_{n}\right\| & \leq \sum_{j=n}^{m+n-1}\left\|x_{j+1}-x_{j}\right\| \\
& \leq \sum_{j=n}^{m+n-1}\left(1+q b_{j}\right)\left\|y_{j}-x_{j}\right\| \\
& \leq\left(1+q b_{0}\right) \sum_{j=n}^{m+n-1} \prod_{i=0}^{j-1} \theta_{i}\left\|y_{0}-x_{0}\right\| \\
& \leq\left(1+q b_{0}\right) \sum_{j=n}^{m+n-1} \prod_{i=0}^{j-1} \xi^{\frac{(2 p+1)^{i}-1}{p}} \theta\left\|y_{0}-x_{0}\right\| \\
& \leq\left(1+q b_{0}\right) \sum_{j=n}^{m+n-1} \prod_{i=0}^{j-1} \xi^{\frac{(2 p+1)^{i}}{p}} \gamma_{0}^{-\frac{1}{p}}\left\|y_{0}-x_{0}\right\| \\
& =\left(1+q b_{0}\right) \sum_{j=n}^{m+n-1} \sum^{j-1} \frac{(2 p+1)^{i}}{p} \gamma_{0}^{-\frac{1}{p}}\left\|y_{0}-x_{0}\right\| \\
& \leq\left(1+q b_{0}\right)\left(\sum_{j=n}^{m+n-1} \xi^{\frac{(2 p+1)^{j}-1}{2 p^{2}}} \gamma_{0}^{-\frac{j}{p}}\right)\left\|y_{0}-x_{0}\right\| .
\end{aligned}
$$

By Bernoulli's inequality, for each $j \geq 0$ and $y>-1$, we have $(1+y)^{j} \geq 1+j y$. Hence we have

$$
\begin{aligned}
& \left\|x_{m+n}-x_{n}\right\| \\
\leq & \left(1+q b_{0}\right) \xi^{-\frac{1}{2 p^{2}}} \gamma_{0}^{-\frac{n}{p}}\left(\xi^{\frac{(2 p+1)^{n}}{2 p^{2}}}+\xi^{\frac{(2 p+1)^{n}(2 p+1)}{2 p^{2}}} \gamma_{0}^{-\frac{1}{p}}+\cdots+\xi^{\frac{(2 p+1)^{n}(2 p+1)^{m-1}}{2 p^{2}}} \gamma_{0}^{-\frac{(m-1)}{p}}\right) \eta \\
\leq & \left(1+q b_{0}\right) \xi^{-\frac{1}{2 p^{2}}} \gamma_{0}^{-\frac{n}{p}}\left(\xi^{\frac{(2 p+1)^{n}}{2 p^{2}}}+\xi^{\frac{(2 p+1)^{n}(1+2 p)}{2 p^{2}}} \gamma_{0}^{-\frac{1}{p}}+\cdots+\xi^{\frac{(2 p+1)^{n}(1+2(m-1) p)}{2 p^{2}}} \gamma_{0}^{-\frac{(m-1)}{p}}\right) \eta \\
= & \left(1+q b_{0}\right) \xi^{-\frac{1}{2 p^{2}}} \gamma_{0}^{-\frac{n}{p}}\left(\xi^{\frac{(2 p+1)^{n}}{2 p^{2}}}+\xi^{(2 p+1)^{n}\left(\frac{1}{2 p^{2}}+\frac{1}{p}\right)} \gamma_{0}^{-\frac{1}{p}}+\cdots+\xi^{(2 p+1)^{n}\left(\frac{1}{2 p^{2}}+\frac{m-1}{p}\right)} \gamma_{0}^{-\frac{(m-1)}{p}}\right) \eta \\
= & \left(1+q b_{0}\right) \xi^{\frac{(2 p+1)^{n}-1}{2 p^{2}}} \gamma_{0}^{-\frac{n}{p}}\left(1+\left(\xi^{(2 p+1)^{n}} \gamma_{0}^{-1}\right)^{\frac{1}{p}}+\cdots+\left(\xi^{(2 p+1)^{n}} \gamma_{0}^{-1}\right)^{\frac{m-1}{p}}\right) \eta \\
= & \left(1+q b_{0}\right) \xi^{\frac{(2 p+1)^{n}-1}{2 p^{2}}} \gamma_{0}^{-\frac{n}{p}}\left(\frac{1-\left(\xi^{(2 p+1)^{n}} \gamma_{0}^{-1}\right)^{\frac{m}{p}}}{1-\left(\xi^{(2 p+1)^{n}} \gamma_{0}^{-1}\right)^{\frac{1}{p}}}\right) \eta .
\end{aligned}
$$

Since the sequence $\left\{x_{n}\right\}$ is a Cauchy sequence and hence it converges to some point $x^{*} \in B_{r}\left[x_{0}\right]$. From Equations (13), (C2), and (15), we have

$$
\begin{aligned}
\left\|L F\left(x_{n}\right)\right\| & \leq\left\|z_{n}-y_{n}\right\|+\left\|y_{n}-x_{n}\right\| \\
& \leq k\left\|y_{n}-x_{n}\right\|+\left\|y_{n}-x_{n}\right\| \\
& \leq(1+k) \theta^{n} \eta .
\end{aligned}
$$


Taking the limit as $n \rightarrow \infty$ and using the continuity of $F$ and the linearity of $L$, we have

$$
F\left(x^{*}\right)=0 .
$$

(3) Taking the limit as $m \rightarrow \infty$ in Equation (22), we have

$$
\left\|x^{*}-x_{n}\right\| \leq \frac{\left(1+q b_{0}\right) \eta}{\xi^{1 / 2 p^{2}}\left(1-\xi^{\frac{(2 p+1)^{n}}{p}} \gamma_{0}^{-\frac{1}{p}}\right) \gamma_{0}^{n / p}}\left(\xi^{1 / 2 p^{2}}\right)^{(2 p+1)^{n}}
$$

for each $n \in \mathbb{N}_{0}$.

(4) Here we prove

$$
\frac{\left\|x_{n+1}-x^{*}\right\|}{\left\|x_{n}-x^{*}\right\|^{2 p+1}} \leq K
$$

for all $n \in \mathbb{N}_{0}$ and for some $K>0$. One can easily observe that there exists $n_{0}>0$ such that

$$
\left\|x_{n}-x^{*}\right\|<1
$$

whenever $n \geq n_{0}$. Using Equations (13) and (24), we have

$$
\begin{aligned}
\left\|z_{n}-x^{*}\right\| & =\left\|x_{n}-x^{*}-\operatorname{LF}\left(x_{n}\right)\right\| \\
& =\left\|x_{n}-x^{*}-L\left(F\left(x_{n}\right)-F\left(x^{*}\right)\right)\right\| \\
& =\left\|x_{n}-x^{*}-L \int_{0}^{1} F_{x^{*}+t\left(x_{n}-x^{*}\right)}^{\prime}\left(x_{n}-x^{*}\right) d t\right\| \\
& \leq \int_{0}^{1}\left\|I-L F_{x^{*}+t\left(x_{n}-x^{*}\right)}^{\prime}\right\|\left\|x_{n}-x^{*}\right\| d t \\
& \leq k\left\|x_{n}-x^{*}\right\|
\end{aligned}
$$

and

$$
\begin{aligned}
\left\|y_{n}-x^{*}\right\| & =\left\|x_{n}-x^{*}-F_{z_{n}}^{\prime-1} F\left(x_{n}\right)\right\| \\
& =\left\|F_{z_{n}}^{\prime-1}\left[F_{z_{n}}^{\prime}\left(x_{n}-x^{*}\right)-F\left(x_{n}\right)\right]\right\| \\
& \leq\left\|F_{z_{n}}^{\prime-1}\right\|\left\|F\left(x_{n}\right)-x^{*}-F_{z_{n}}^{\prime}\left(x_{n}-x^{*}\right)\right\| \\
& =\left\|F_{z_{n}}^{-1}\right\|\left\|\int_{0}^{1}\left(F_{x^{*}+t\left(x_{n}-x^{*}\right)}^{\prime}-F_{z_{n}}^{\prime}\right)\left(x_{n}-x^{*}\right)\right\| d t \\
& \leq\left\|F_{z_{n}}^{\prime-1}\right\| \int_{0}^{1}\left\|F_{x^{*}+t\left(x_{n}-x^{*}\right)}^{\prime}-F_{z_{n}}^{\prime}\right\|\left\|x_{n}-x^{*}\right\| d t \\
& \leq\left\|F_{z_{n}}^{-1}\right\| \int_{0}^{1}\left(\left\|F_{x^{*}+t\left(x_{n}-x^{*}\right)}^{\prime}-F_{x^{*}}^{\prime}\right\|+\left\|F_{x^{*}}^{\prime}-F_{z_{n}}^{\prime}\right\|\right)\left\|x_{n}-x^{*}\right\| d t \\
& \leq\left\|F_{z_{n}}^{\prime-1}\right\| \int_{0}^{1}\left(\omega\left(t\left\|x_{n}-x^{*}\right\|\right)+\omega\left(\left\|z_{n}-x^{*}\right\|\right)\right)\left\|x_{n}-x^{*}\right\| d t \\
& \leq\left\|F_{z_{n}}^{\prime-1}\right\| \int_{0}^{1}\left(t^{p}\left\|x_{n}-x^{*}\right\|^{p} \omega(1)+\omega\left(k\left\|x_{n}-x^{*}\right\|\right)\right)\left\|x_{n}-x^{*}\right\| d t \\
& \leq\left\|F_{z_{n}}^{\prime-1}\right\|\left(\frac{1}{p+1}+k^{p}\right) \omega(1)\left\|x_{n}-x^{*}\right\|^{p+1} \\
& =\left\|F_{z_{n}}^{\prime-1}\right\| q \omega(1)\left\|x_{n}-x^{*}\right\|^{p+1} .
\end{aligned}
$$

Using Equations (13), (24) and (25), we have 


$$
\begin{aligned}
& \left\|x_{n+1}-x^{*}\right\| \\
= & \left\|y_{n}-F_{z_{n}}^{\prime-1} F\left(y_{n}\right)-x^{*}\right\| \\
\leq & \left\|F_{z_{n}}^{\prime-1}\right\|\left\|F\left(y_{n}\right)-F\left(x^{*}\right)-F_{z_{n}}^{\prime}\left(y_{n}-x^{*}\right)\right\| \\
= & \left\|F_{z_{n}}^{\prime-1}\right\|\left\|\int_{0}^{1}\left(F_{x^{*}+t\left(y_{n}-x^{*}\right)}^{\prime}-F_{z_{n}}^{\prime}\right)\left(y_{n}-x^{*}\right)\right\| d t \\
\leq & \left\|F_{z_{n}}^{\prime-1}\right\| \int_{0}^{1}\left\|F_{x^{*}+t\left(y_{n}-x^{*}\right)}^{\prime}-F_{z_{n}}^{\prime}\right\|\left\|y_{n}-x^{*}\right\| d t \\
= & \left\|F_{z_{n}}^{\prime-1}\right\| \int_{0}^{1}\left(\left\|F_{x^{*}+t\left(y_{n}-x^{*}\right)}^{\prime}-F_{x^{*}}^{\prime}\right\|+\left\|F_{x^{*}}^{\prime}-F_{z_{n}}^{\prime}\right\|\right)\left\|y_{n}-x^{*}\right\| d t \\
\leq & \left\|F_{z_{n}}^{\prime-1}\right\| \int_{0}^{1}\left(\omega\left(t\left\|y_{n}-x^{*}\right\|\right)+\omega\left(k\left\|x_{n}-x^{*}\right\|\right)\right)\left\|y_{n}-x^{*}\right\| d t \\
= & \left\|F_{z_{n}}^{\prime-1}\right\| \int_{0}^{1}\left(t^{p} \omega\left(\left\|F_{z_{n}}^{\prime-1}\right\| q \omega(1)\left\|x_{n}-x^{*}\right\|^{p+1}\right)+\omega\left(k\left\|x_{n}-x^{*}\right\|\right)\right) d t \\
& \times\left\|F_{z_{n}}^{\prime-1}\right\| q \omega(1)\left\|x_{n}-x^{*}\right\|^{p+1} \\
\leq & \left\|F_{z_{n}}^{\prime-1}\right\|^{2} \int_{0}^{1}\left(t^{p}\left\|x_{n}-x^{*}\right\|^{p(p+1)} \omega\left(\left\|F_{z_{n}}^{\prime-1}\right\| q \omega(1)\right)+k^{p}\left\|x_{n}-x^{*}\right\|^{p} \omega(1)\right) d t \\
& \times q \omega(1)\left\|x_{n}-x^{*}\right\|^{p+1} \\
= & \left\|F_{z_{n}}^{\prime-1}\right\|^{2}\left(\frac{\left\|x_{n}-x^{*}\right\|^{p^{2}} \omega\left(\left\|F_{z_{n}}^{\prime-1}\right\| q \omega(1)\right)}{p+1}+k^{p}\right) q \omega(1)\left\|x_{n}-x^{*}\right\|^{2 p+1} \\
= & K_{n}\left\|x_{n}-x^{*}\right\|^{2 p+1},
\end{aligned}
$$

where

$$
K_{n}=\left\|F_{z_{n}}^{\prime-1}\right\|^{2}\left(\frac{\left\|x_{n}-x^{*}\right\|^{p^{2}} \omega\left(\left\|F_{z_{n}}^{\prime-1}\right\| q \omega(1)\right)}{p+1}+k^{p}\right) q \omega(1) .
$$

Let $\left\|F_{x^{*}}^{\prime-1}\right\| \leq d$ and $0<d<\omega(\sigma)^{-1}$, where $\sigma>0$. Then, for all $x \in B_{\sigma}\left(x^{*}\right)$, we have

$$
\left\|I-F_{x^{*}}^{\prime-1} F_{x}^{\prime}\right\| \leq\left\|F_{x^{*}}^{\prime-1}\right\|\left\|F_{x^{*}}^{\prime}-F_{x}^{\prime}\right\| \leq d \omega(\sigma)<1
$$

and so, by Lemma 1, we have

$$
\left\|F_{x}^{\prime-1}\right\| \leq \frac{d}{1-d \omega(\sigma)}:=\lambda
$$

Since $x_{n} \rightarrow x^{*}$ and $z_{n} \rightarrow x^{*}$ as $n \rightarrow \infty$, there exists a positive integer $N_{0}$ such that

$$
\left\|F_{z_{n}}^{\prime-1}\right\| \leq \frac{d}{1-d \omega(\sigma)}
$$

for all $n \geq N_{0}$. Thus, for all $n \geq N_{0}$, one can easily observe that

$$
K_{n} \leq \lambda^{2}\left(\frac{\sigma^{p^{2}} \omega(\lambda q \omega(1))}{p+1}+k^{p}\right) q \omega(1)=K .
$$

This shows that the $R$-order of convergence at least $(2 p+1)$. This completes the proof.

\section{Applications}

\subsection{Fixed Points of Smooth Operators}

Let $X$ be a Banach spaces and $D$ be a nonempty open convex subset of $X$. Let $G: D \subset X \rightarrow X$ be a nonlinear operator such that $D$ is Fréchet differentiable at each point of $D$ and let $L \in B(X, X)$ such that $(I-L(I-G))(D) \subseteq D$. For $F=I-G$, the Newton-like algorithm Equation (13) reduces to the following Stirling-like method for computing fixed point of the operator $G$ : 
Starting with $x_{0} \in D$ and after $x_{n} \in D$ is defined, we define the next iterate $x_{n+1}$ as follows:

$$
\left\{\begin{array}{l}
z_{n}=(I-L(I-G))\left(x_{n}\right), \\
y_{n}=\left(I-\left(I-G_{z_{n}}^{\prime}\right)^{-1}(I-G)\right)\left(x_{n}\right), \\
x_{n+1}=\left(I-\left(I-G_{z_{n}}^{\prime}\right)^{-1}(I-G)\right)\left(y_{n}\right)
\end{array}\right.
$$

for each $n \in \mathbb{N}_{0}$.

For the choice of $X=Y$ and $F=I-G$, Theorem 1 reduces to the following:

Theorem 2. Let $D$ be a nonempty open convex subset of a Banach space $X, G: D \rightarrow X$ be a Fréchet differentiable at each point of $D$ with values into itself. Let $L \in B(X)$ be such that $(I-L(I-G))(D) \subseteq D$. Let $x_{0} \in D$ be such that $z_{0}=x_{0}-L\left(x_{0}-G\left(x_{0}\right)\right)$ and let $\left(I-G_{z_{0}}^{\prime}\right)^{-1} \in B(X)$ exist. Let $\omega \in \Phi$ and $\alpha$ be $a$ solution of the Equation (9). Assume that the conditions (C5)-(C6) and the following conditions hold:

(C7) $\left\|\left(I-G_{z_{0}}^{\prime}\right)^{-1}\right\| \leq \beta$ for some $\beta>0$;

(C8) $\left\|\left(I-G_{z_{0}}^{\prime}\right)^{-1}\left(x_{0}-G\left(x_{0}\right)\right)\right\| \leq \eta$ for some $\eta>0$;

(C9) $\left\|G_{x}^{\prime}-G_{y}^{\prime}\right\| \leq \omega(\|x-y\|)$ for all $x, y \in D$;

(C10) $\left\|I-L\left(I-G_{x}^{\prime}\right)\right\| \leq k$ for all $x \in D$ and for some $k \in\left(0, \frac{1}{3}\right]$.

Then the sequence $\left\{x_{n}\right\}$ generated by Equation (26) is well defined, remains in $B_{r}\left[x_{0}\right]$ and converges to the fixed point $x^{*} \in B_{r}\left[x_{0}\right]$ of the operator $G$ and the sequence $\left\{x_{n}\right\}$ has $R$-order of convergence at least $2 p+1$.

We give an example to illustrate Theorem 2.

Example 1. Let $X=Y=\mathbb{R}$ and $D=(-1,1) \subset X$. Define a mapping $G: D \rightarrow \mathbb{R}$ by

$$
G(x)=\frac{1.1 x^{3}-x}{6}
$$

for all $x \in D$. Define $L: \mathbb{R} \rightarrow \mathbb{R}$ by $L(x)=\frac{7.9}{7} x$ for all $x \in \mathbb{R}$. One can easily observe that

$$
(I-L(I-G))(x) \in D
$$

for all $x \in D$. Clearly, $G$ is differentiable on $D$ and its derivative at $x \in D$ is $G_{x}^{\prime}=\frac{3.3 x^{2}-1}{6}$ and $G_{x}^{\prime}$ is bounded with $\left\|G_{x}^{\prime}\right\| \leq 0.3833$ for all $x \in D$ and $G^{\prime}$ satisfies the Lipschitz condition

$$
\left\|G_{x}^{\prime}-G_{y}^{\prime}\right\| \leq K\|x-y\|
$$

for all $x, y \in D$, where $K=1.1$. For $x_{0}=0.3$, we have

$$
\begin{gathered}
z_{0}=(I-L(I-G))\left(x_{0}\right)=-0.0894135714, \quad\left\|\left(I-G_{z_{0}}^{\prime}\right)^{-1}\right\| \leq 0.860385626=\beta, \\
\left\|\left(I-G_{z_{0}}^{\prime}\right)^{-1}\left(x_{0}-G\left(x_{0}\right)\right)\right\| \leq 0.29687606=\eta .
\end{gathered}
$$

For $p=1, q=\frac{5}{6}$ and $\omega(t)=K t$ for all $t \geq 0$, we have

$$
\begin{gathered}
b_{0}=\beta \omega(\eta)=0.280970684<1, \\
\theta=\frac{\left(\frac{q^{p} b_{0}^{p}}{p+1}+k^{p}\right) q b_{0}^{2}}{1-k\left(1+q b_{0}\right) b_{0}}=0.0335033167<1
\end{gathered}
$$

and $r=0.563139828$. Hence all the conditions of Theorem 2 are satisfied. Therefore, the sequence $\left\{x_{n}\right\}$ generated by Equation (26) is in $B_{r}\left[x_{0}\right]$ and it converges to the fixed point $x^{*}=0 \in B_{r}\left[x_{0}\right]$ of $G$. 
Remark 1. In Example 1, $\left\|G_{x}^{\prime}\right\| \leq 0.38333>\frac{1}{3}$. Thus the condition $(\Omega)$ does not hold and so we can not apply Parhi and Gupta [21] (Theorem 1) for finding fixed points of the operators like G defined by (27). Thus the Stirling-like method defined by Equation (26) provides an affirmative answer of the Problem 1.

If the condition $(\Omega)$ holds, then Theorem 2 with $L=I$ reduces to the main result of Parhi and Gupta [21] as follows:

Corollary 1. [21] (Theorem 1) Let $D$ be a nonempty open convex subset of a Banach space $X$ and $G: D \rightarrow D$ be a Fréchet differentiable operator and let $x_{0} \in D$ with $z_{0}=G\left(x_{0}\right)$. Let $\left(I-G_{z_{0}}^{\prime}\right)^{-1} \in B(X)$ exists and $\omega \in \Phi$. Assume that the conditions (C5)-(C9) and the following condition holds:

(C11) $\left\|G_{x}^{\prime}\right\| \leq k$ for all $x \in D$ and for some $k \in\left(0, \frac{1}{3}\right]$.

Then the sequence $\left\{x_{n}\right\}$ generated by Equation (8) is well defined, remains in $B_{r}\left[x_{0}\right]$ and converges to the fixed point $x^{*} \in B_{r}\left[x_{0}\right]$ of the operator $G$ with $R$-order of the convergence at least $2 p+1$.

Example 2. Let $X=Y=\mathbb{R}$ and $D=(-6,6) \subset X$. Define a mapping $G: D \rightarrow \mathbb{R}$ by

$$
G(x)=2+e^{\frac{\sin x}{5}}
$$

for all $x \in D$. It is obvious that $G$ is Fréchet differentiable on $D$ and its Fréchet derivative at $x \in D$ is $G_{x}^{\prime}=\frac{\cos x}{5} e^{\frac{\sin x}{5}}$. Clearly, $G_{x}^{\prime}$ is bounded with $\left\|G_{x}^{\prime}\right\| \leq 0.22<\frac{1}{3}=k$ and

$$
\left\|G_{x}^{\prime}-G_{y}^{\prime}\right\| \leq K\|x-y\|
$$

for all $x, y \in D$, where $K=0.245$. For $x_{0}=0$, we have

$$
z_{0}=G\left(x_{0}\right)=3, \quad\left\|\left(I-G_{z_{0}}^{\prime}\right)^{-1}\right\| \leq 0.834725586524139=\beta
$$

and

$$
\left\|\left(I-G_{z_{0}}^{\prime}\right)^{-1}\left(x_{0}-G\left(x_{0}\right)\right)\right\| \leq 2.504176759572418=\eta .
$$

For $p=1, q=\frac{5}{6}$ and $\omega(t)=K t$ for all $t \geq 0$, we have

$$
\begin{gathered}
b_{0}=\beta K \eta=0.512123601526580<1, \\
\theta=\frac{\left(\frac{q^{p} b_{0}^{p}}{p+1}+k^{p}\right) q b_{0}^{2}}{1-k\left(1+q b_{0}\right) b_{0}}=0.073280601270728<1
\end{gathered}
$$

and $r=5.147038576039456$.

Hence all the conditions of Theorem 2 with $L=I$ are satisfied. Therefore, the sequence $\left\{x_{n}\right\}$ generated by Equation (26) is in $B_{r}\left[x_{0}\right]$ and it converges to the fixed point $x^{*}=3.023785446275295 \in B_{r}\left[x_{0}\right]$ of $G$ (Table 1).

Table 1. A priori error bounds.

\begin{tabular}{lc}
\hline$n$ & $\left\|x_{n}-x^{*}\right\|$ \\
\hline 0 & 3.0237854462752 \\
1 & $1.7795738211156 \times 10^{-2}$ \\
2 & $6.216484249588206 \times 10^{-6}$ \\
3 & $2.335501569916687 \times 10^{-9}$ \\
4 & $8.775202786637237 \times 10^{-13}$ \\
5 & $4.440892098500626 \times 10^{-16}$ \\
\hline
\end{tabular}




\subsection{Fredholm Integral Equations}

Let $X$ be a Banach space over the field $\mathbb{F}(\mathbb{R}$ or $\mathbb{C}$ ) with the norm $\|\cdot\|$ and $D$ be an open convex subset of $X$. Further, let $B(X)$ be the Banach space of bounded linear operators from $X$ into itself. Let $S \in B(X), u \in X$ and $\lambda \in \mathbb{F}$. We investigate a solution $x \in X$ of the nonlinear Fredholm-type operator equation:

$$
x-\lambda S Q(x)=u,
$$

where $Q: D \rightarrow X$ is continuously Fréchet differentiable on $D$. The operator Equation (28) has been discussed in $[10,38,39]$. Define an operator $F: D \rightarrow X$ by

$$
F(x)=x-\lambda S Q(x)-u
$$

for all $x \in D$. Then solving the operator Equation (29) is equivalent to solving the operator Equation (1). From Equation (29), we have

$$
F_{x}^{\prime}(h)=h-\lambda S Q_{x}^{\prime}(h)
$$

for all $h \in X$. Now, we apply Theorem 1 to solve the operator Equation (28).

Theorem 3. Let $X$ be a Banach space and $D$ an open convex subset of $X$. Let $Q: D \rightarrow X$ be a continuously Fréchet differentiable mapping at each point of $D$. Let $L, S \in B(X)$ and $u \in X$. Assume that, for any $x_{0} \in D$, $z_{0}=x_{0}-L\left(x_{0}-\lambda S Q\left(x_{0}\right)-u\right)$ and $\left(I-\lambda S Q_{z_{0}}^{\prime}\right)^{-1}$ exist. Assume that the condition (C6) and the following conditions hold:

(C12) $(I-L(I-\lambda S Q))(x)-u \in D$ for all $x \in D$;

(C13) $\left\|\left(I-\lambda S Q_{z_{0}}^{\prime}\right)^{-1}\right\| \leq \beta$ for some $\beta>0$;

(C14) $\left\|\left(I-\lambda S Q_{z_{0}}^{\prime}\right)^{-1}\left(x_{0}-\lambda S Q\left(x_{0}\right)-u\right)\right\| \leq \eta$ for some $\eta>0$;

(C15) $\left\|Q_{x}^{\prime}-Q_{y}^{\prime}\right\| \leq \omega_{0}(\|x-y\|)$ for all $x, y \in D$, where $\omega_{0} \in \Phi$;

(C16) $\omega_{0}(s t) \leq s^{p} \omega_{0}(t), s \in[0,1]$ and $t \in[0, \infty)$;

(C17) $\left\|I-L\left(I-\lambda S Q_{x}^{\prime}\right)\right\| \leq k, k \leq \frac{1}{3}$ for all $x \in D$.

Then we have the following:

(1) The sequence $\left\{x_{n}\right\}$ generated by

$$
\left\{\begin{array}{l}
z_{n}=x_{n}-L\left(x_{n}-\lambda S Q\left(x_{n}\right)-u\right) \\
y_{n}=x_{n}-\left(I-\lambda S Q_{z_{n}}^{\prime}\right)^{-1}\left(x_{n}-\lambda S Q\left(x_{n}\right)-u\right) \\
x_{n+1}=y_{n}-\left(I-\lambda S Q_{z_{n}}^{\prime}\right)^{-1}\left(y_{n}-\lambda S Q\left(y_{n}\right)-u\right)
\end{array}\right.
$$

for each $n \in \mathbb{N}_{0}$ is well defined, remains in $B_{r}\left[x_{0}\right]$ and converges to a solution $x^{*}$ of the Equation (28).

(2) The R-order convergence of sequence $\left\{x_{n}\right\}$ is at least $2 p+1$.

Proof. Let $F: D \rightarrow X$ be an operator defined by Equation (29). Clearly, $F$ is Fréchet differentiable at each point of $D$ and its Fréchet derivative at $x \in D$ is given by Equation (30). Now, from (C13) and Equation (30), we have $\left\|F_{z_{0}}^{\prime-1}\right\| \leq \beta$ and so it follows that (C3) holds. From (C14), Equations (29) and (30), we have $\left\|F_{z_{0}}^{\prime-1}\left(F\left(x_{0}\right)\right)\right\| \leq \eta$. Hence (C4) is satisfied. For all $x, y \in D$, using (C15), we have

$$
\begin{aligned}
\left\|F_{x}^{\prime}-F_{y}^{\prime}\right\| & =\sup \left\{\left\|\left(F_{x}^{\prime}-F_{y}^{\prime}\right) z\right\|: z \in X,\|z\|=1\right\} \\
& \leq|\lambda|\|S\| \sup \left\{\left\|Q_{x}^{\prime}-Q_{y}^{\prime}\right\|\|z\|: z \in X,\|z\|=1\right\} \\
& \leq|\lambda|\|S\| \omega_{0}(\|x-y\|) \\
& =\omega(\|x-y\|),
\end{aligned}
$$


where $\omega(t)=|\lambda|\|S\| \omega_{0}(t)$. Clearly, $\omega \in \Phi$ and, from (C16), we have

$$
\omega(s t) \leq s^{p} \omega(t)
$$

for all $s \in[0,1]$ and $t \in(0, \infty]$. Thus (C1) and (C5) hold. (C2) follows from (C17) for $c=k \in\left(0, \frac{1}{3}\right]$. Hence all the conditions of Theorem 1 are satisfied. Therefore, Theorem 3 follows from Theorem 1. This completes the proof.

Let $D=X=Y=C[a, b]$ be the space of all continuous real valued functions defined on $[a, b] \subset \mathbb{R}$ with the norm $\|x\|=\sup _{t \in[a, b]}|x(t)|$. Consider, the following nonlinear integral equation:

$$
x(s)=g(s)+\lambda \int_{a}^{b} K(s, t)\left(\mu(x(t))^{1+p}+v(x(t))^{2}\right) d t
$$

for all $s \in[a, b]$ and $p \in(0,1]$, where $g, x \in C[a, b]$ with $g(s) \geq 0$ for all $s \in[a, b], K:[a, b] \times[a, b] \rightarrow \mathbb{R}$ is a continuous nonnegative real-valued function and $\mu, v, \lambda \in \mathbb{R}$. Define two mappings $S, Q: D \rightarrow X$ by

$$
S x(s)=\int_{a}^{b} K(s, t) x(t) d t
$$

for all $s \in[a, b]$ and

$$
Q x(s)=\mu(x(s))^{1+p}+v(x(s))^{2}
$$

for all $\mu, v \in \mathbb{R}$ and $s \in[a, b]$.

One can easily observe that $K$ is bounded on $[a, b] \times[a, b]$, that is, there exists a number $M \geq 0$ such that $|K(s, t)| \leq M$ for all $s, t \in[a, b]$. Clearly, $S$ is bounded linear operator with $\|S\| \leq M(b-a)$ and $Q$ is Fréchet differentiable and its Fréchet derivative at $x \in D$ is given by

$$
Q_{x}^{\prime} h(s)=\left(\mu(1+p) x^{p}+2 v x\right) h(s)
$$

for all $h \in C[a, b]$. For all $x, y \in D$, we have

$$
\begin{aligned}
\left\|Q_{x}^{\prime}-Q_{y}^{\prime}\right\| & =\sup \left\{\left\|\left(Q_{x}^{\prime}-Q_{y}^{\prime}\right) h\right\|: h \in C[a, b],\|h\|=1\right\} \\
& \leq \sup \left\{\left\|\left(\mu(1+p)\left(x^{p}-y^{p}\right)+2 v(x-y)\right) h\right\|: h \in C[a, b],\|h\|=1\right\} \\
& \leq \sup \left\{\left(|\mu|(1+p)\left\|x^{p}-y^{p}\right\|+2|v|\|x-y\|\right)\|h\|: h \in C[a, b],\|h\|=1\right\} \\
& \leq|\mu|(1+p)\|x-y\|^{p}+2|v|\|x-y\| \\
& =\omega_{0}(\|x-y\|),
\end{aligned}
$$

where $\omega_{0}(t)=|\mu|(1+p) t^{p}+2|v| t, t \geq 0$ with

$$
\omega_{0}(s t) \leq s^{p} \omega_{0}(t)
$$

for all $s \in[0,1]$ and $t \in[0, \infty)$. For any $x \in D$, using Equations (33) and (35), we have

$$
\begin{aligned}
& \left\|S Q_{x}^{\prime}\right\| \\
= & \sup \left\{\left\|S Q_{x}^{\prime} h\right\|: h \in X,\|h\|=1\right\} \\
= & \sup \left\{\sup _{s \in[a, b]}\left|\int_{a}^{b} K(s, t)\left(\mu(1+p)(x(t))^{p}+2 v x(t)\right) h(t) d t\right|: h \in X,\|h\|=1\right\} \\
\leq & \sup \left\{\int_{a}^{b}|K(s, t)|\left(|\mu|(1+p)|x(t)|^{p}+2|v \| x(t)|\right)|h(t)| d t: h \in X,\|h\|=1\right\} \\
\leq & \left(|\mu|(1+p)\|x\|^{p}+2|v|\|x\|\right) M(b-a)<1 .
\end{aligned}
$$

We now apply Theorem 3 to solve the Fredholm integral Equation (32). 
Theorem 4. Let $D=X=Y=C[a, b]$ and $\mu, v, \lambda, M \in \mathbb{R}$. Let $S, Q: D \rightarrow X$ be operators defined by Equations (33) and (34), respectively. Let $L \in B(X)$ and $x_{0} \in D$ be such that $z_{0}=x_{0}-L\left(x_{0}-\lambda S Q\left(x_{0}\right)-\right.$ $g) \in D$. Assume that the condition (C6) and the following conditions hold:

$\frac{1}{1-|\lambda|\left(|\mu|(1+p)\left\|z_{0}\right\|^{p}+2|v||| z_{0} \|\right) M(b-a)}=\beta$ for some $\beta>0 ;$

(C19) $\frac{\left\|x_{0}-g\right\|+|\lambda|\left(|\mu|\left\|x_{0}\right\|^{p+1}+2|v|\left\|x_{0}\right\|^{2}\right) M(b-a)}{1-|\lambda|\left(|\mu|(1+p)\left\|z_{0}\right\|^{p}+2|v|\left\|z_{0}\right\|\right) M(b-a)}=\eta$ for some $\eta>0$;

(C20) $\|I-L\|+|\lambda|\|L\|\left(|\mu|(1+p)\|x\|^{p}+2|v|\|x\|\right) M(b-a) \leq \frac{1}{3}$ for all $x \in D$.

Then the sequence generated by Equation (31) with $u=g \in X$ is well defined, remains in $B_{r}\left[x_{0}\right]$ and converges to the solution $x^{*} \in B_{r}\left[x_{0}\right]$ of the Equation (32) with R-order convergence at least $(2 p+1)$.

Proof. Note that $D=X=Y=C[a, b]$. Obviously, (C12) holds. Using Equations (C20), (33), (35) and (38), we have

$$
\left\|I-\left(I-\lambda S Q_{z_{0}}^{\prime}\right)\right\| \leq|\lambda|\left(|\mu|(1+p)\left\|z_{0}\right\|^{p}+2|v|\left\|z_{0}\right\|\right) M(b-a)<1 .
$$

Therefore, by Lemma $1,\left(I-\lambda S Q_{z_{0}}^{\prime}\right)^{-1}$ exists and

$$
\left\|\left(I-\lambda S Q_{z_{0}}^{\prime}\right)^{-1}\right\| \leq \frac{1}{1-|\lambda|\left(|\mu|(1+p)\left\|z_{0}\right\|^{p}+2|v|\left\|z_{0}\right\|\right) M(b-a)} .
$$
we have

Hence Equations (C18) and (39) implies (C13) holds. Using Equations (C19), (38) and (39),

$$
\begin{aligned}
& \left\|\left(I-\lambda S Q_{z_{0}}^{\prime}\right)^{-1}\left(x_{0}-\lambda S Q\left(x_{0}\right)-g\right)\right\| \\
\leq & \left\|\left(I-\lambda S Q_{z_{0}}^{\prime}\right)^{-1}\right\|\left(\left\|x_{0}-g\right\|+\left\|\lambda S Q\left(x_{0}\right)\right\|\right) \\
\leq & \frac{\left\|x_{0}-g\right\|+|\lambda|\left(|\mu|\left\|x_{0}\right\|^{p+1}+2|v|\left\|x_{0}\right\|^{2}\right) M(b-a)}{1-|\lambda|\left(|\mu|(1+p)\left\|z_{0}\right\|^{p}+2|v|\left\|z_{0}\right\|\right) M(b-a)} \\
\leq & \eta .
\end{aligned}
$$

Thus the condition (C14) is satisfied. The conditions (C15) and (C16) follow from Equations (36) and (37), respectively. Now, from Equation (C20) and (38), we have

$$
\begin{aligned}
\left\|I-L\left(I-\lambda S Q_{x}^{\prime}\right)\right\| & \leq\|I-L\|+\|L\|\left\|\lambda S Q_{x}^{\prime}\right\| \\
& \leq\|I-L\|+\|L\||\lambda|\left(|\mu|(1+p)\|x\|^{p}+2|v|\|x\|\right) M(b-a) \\
& \leq \frac{1}{3} .
\end{aligned}
$$

This implies that (C17) holds. Hence all the conditions of Theorem 3 are satisfied. Therefore, Theorem 4 follows from Theorem 3. This completes the proof.

Now, we give one example to illustrate Theorem 3.

Example 3. Let $X=Y=C[0,1]$ be the space of all continuous real valued functions defined on $[0,1]$. Let $D=\left\{x: x \in C[0,1],\|x\|<\frac{3}{2}\right\} \subset C[0,1]$. Consider the following nonlinear integral equation:

$$
x(s)=\sin (\pi s)+\frac{1}{10} \int_{0}^{1} \cos (\pi s) \sin (\pi t)(x(t))^{p+1} d t, p \in(0,1] .
$$

Define two mappings $S: X \rightarrow X$ and $Q: D \rightarrow Y$ by

$$
S(x)(s)=\int_{0}^{1} K(s, t) x(t) d t, \quad Q(x)(s)=(x(s))^{p+1},
$$


where $K(s, t)=\cos (\pi s) \sin (\pi t)$. For $u=\sin (\pi s)$, the problem Equation (40) is equivalent to the problem Equation (28). Here, one can easily observe that $S$ is bounded linear operator with $\|S\| \leq 1$ and $Q$ is Fréchet differentiable with $Q_{x}^{\prime} h(s)=(p+1)(x(s))^{p} h(s)$ for all $h \in X$ and $s \in[0,1]$. For all $x, y \in D$, we have

$$
\left\|Q_{x}^{\prime}-Q_{y}^{\prime}\right\| \leq(p+1)\|x-y\|^{p}=\omega_{0}(\|x-y\|),
$$

where $\omega_{0}(t)=(p+1) t^{p}$ for any $t \geq 0$. Clearly, $\omega_{0} \in \Phi$. Define a mapping $F: D \rightarrow X$ by

$$
F(x)(s)=x(s)-\frac{1}{10} S Q(x)(s)-\sin (\pi s) .
$$

Clearly, $F$ is Fréchet differentiable on $D$. We now show that (C12) holds for $L=I \in B(X)$. Note that

$$
\|(I-L(I-\lambda S Q))(x)-u\|=\left\|\frac{1}{10} S Q(x)(s)+\sin (\pi s)\right\| \leq \frac{1}{10}\left(\frac{3}{2}\right)^{p+1}+1<\frac{3}{2}
$$

for all $x \in D$. Thus $(I-L(I-\lambda S Q))(x)-u \in D$ for all $x \in D$. For all $x \in D$, we have

$$
\left\|I-F_{x}^{\prime}\right\| \leq \frac{p+1}{10}\|x\|^{p} \leq \frac{p+1}{10}\left(\frac{3}{2}\right)^{p}=k .
$$

Therefore, by Lemma $1, F_{x}^{\prime-1}$ exists and

$$
F_{x}^{\prime-1} U(s)=U(s)+\frac{(p+1) \cos (\pi s) \int_{0}^{1} \sin (\pi t)(x(t))^{p} U(t) d t}{10-(p+1) \int_{0}^{1} \sin (\pi t) \cos (\pi t)(x(t))^{p} d t}
$$

for all $U \in Y$.

Let $x_{0}(s)=\sin (\pi s), \omega(t)=\frac{\omega_{0}(t)}{10}=\frac{p+1}{10} t^{p}$. Then we have the following:

(a) $x_{0} \in X, F\left(x_{0}(s)\right)=-\frac{\cos (\pi s)}{10} \int_{0}^{1}(\sin (\pi t))^{p+2} d t$;

(b) $z_{0}(s)=x_{0}(s)-F\left(x_{0}(s)\right)=\sin (\pi s)+\frac{\cos (\pi s)}{10} \int_{0}^{1}(\sin (\pi t))^{p+2} d t$;

(c) $\left\|F_{z_{0}}^{\prime-1}\right\| \leq \frac{10^{p+1}}{10^{p+1}-(p+1) 11^{p}}=\beta$;

(d) $\left\|F_{z_{0}}^{\prime-1} F\left(x_{0}\right)\right\| \leq \frac{10^{p}}{10^{p+1}-(p+1) 11^{p}}=\eta$;

(e) $b_{0}=\beta \omega(\eta)=\frac{(p+1) 10^{p(p+1)}}{\left(10^{p+1}-(p+1) 11^{p}\right)^{p+1}}$ and $q=\frac{1}{p+1}+\left(\frac{p+1}{10}\right)^{p}\left(\frac{3}{2}\right)^{p^{2}}$.

One can easily observe that $\theta=\frac{\left(\frac{q b_{0}}{2}+k\right) q b_{0}{ }^{2}}{1-k(1+q) b_{0}}<1$ for all $p \in(0,1]$ and $r=\frac{\left(1+q b_{0}\right) \eta}{1-\theta}$. Hence all the conditions of Theorem 3 are satisfied. Therefore, the sequence $\left\{x_{n}\right\}$ generated by Equation (31) is well defined, remains in $B_{r}\left[x_{0}\right]$ and converges to a solution of the integral Equation (40).

For $p=1$, the convergence behavior of Newton-like method Equation (31) is given in Table 2.

Table 2. Iterates of Newton-like method Equation (31).

\begin{tabular}{cccc}
\hline$n$ & $x_{n}(s)$ & $z_{n}(s)$ & $y_{n}(s)$ \\
\hline 0 & $\sin (\pi s)$ & $\sin (\pi s)+0.0424413182 \cos (\pi s)$ & $\sin (\pi s)+0.0425947671 \cos (\pi s)$ \\
1 & $\sin (\pi s)+0.0424794035 \cos (\pi s)$ & $\sin (\pi s)+0.0424791962 \cos (\pi s)$ & $\sin (\pi s)+0.0424796116 \cos (\pi s)$ \\
2 & $\sin (\pi s)+0.0424795616 \cos (\pi s)$ & $\sin (\pi s)+0.042479611 \cos (\pi s)$ & $\sin (\pi s)+0.0424796112 \cos (\pi s)$ \\
3 & $\sin (\pi s)+0.0424796111 \cos (\pi s)$ & $\sin (\pi s)+0.0424796109 \cos (\pi s)$ & $\sin (\pi s)+0.0424796113 \cos (\pi s)$ \\
\hline
\end{tabular}

\section{Conclusions}

The semilocal convergence of the third order Newton-like method for finding zeros of an operator from a Banach space to another Banach space and the corresponding Stirling-like method for finding 
fixed points of an operator on a Banach space are established under the $\omega$-continuity condition. Our iterative technique is applied to nonlinear Fredholm-type operator equations. The $R$-order of our methods are clearly shown to be equal to at least $2 p+1$ for any $p \in(0,1]$. Some numerical examples are given in support of our work, where earlier work cannot apply. In future, our iterative techniques can be applied in optimization problems.

Author Contributions: The authors have contributed equally to this paper.

Funding: This research has received no exteranl funding.

Acknowledgments: We would like to thank the reviewers for their valuable suggestions for improvement of this paper.

Conflicts of Interest: The authors declare no conflict of interest.

\section{Reference}

1. Kantorovich, L.V.; Akilov, G.P. Functional Analysis; Aregamon Press: Oxford, UK, 1982.

2. Kantorovich, L.V. On Newton's method for functional equations. Dokl. Akad. Nauk. SSSR 1948, 59, 1237-1240. (In Russian)

3. Rheinbolt, W.C. A unified Convergence theory for a class of iterative processes. SIAM J. Numer. Anal. 1968, 5, 42-63. [CrossRef]

4. Argyros, I.K.; Cho, Y.J.; Hilout, S. Numerical Methods for Equations and its Applications; CRC Press/Taylor \& Francis Group Publ. Comp.: New York, NY, USA, 2012.

5. Argyros, I.K.; Hilout, S. Improved generaliged differentiability conditions for Newton-like methods. J. Complex. 2010, 26, 316-333. [CrossRef]

6. Argyros, I.K.; Hilout, S. Majorizing sequences for iterative methods. J. Comput. Appl. Math. 2012, 236, 1947-1960. [CrossRef]

7. Argyros, I.K. An improved error analysis for Newton-like methods under generalized conditions. J. Comput. Appl. Math. 2003, 157, 169-185. [CrossRef]

8. Argyros, I.K.; Hilout, S. On the convergence of Newton-type methods under mild differentiability conditions. Number. Algorithms 2009, 52, 701-726. [CrossRef]

9. Sahu, D.R.; Singh, K.K.; Singh, V.K. Some Newton-like methods with sharper error estimates for solving operator equations in Banach spaces. Fixed Point Theory Appl. 2012, 78, 1-20. [CrossRef]

10. Sahu, D.R.; Singh, K.K.; Singh, V.K. A Newton-like method for generalized operator equations in Banach spaces. Numer. Algorithms 2014, 67, 289-303. [CrossRef]

11. Sahu, D.R.; Cho, Y.J.; Agarwal, R.P.; Argyros, I.K. Accessibility of solutions of operator equations by Newton-like Methods. J. Comlex. 2015, 31, 637-657. [CrossRef]

12. Argyros, I.K. A unifying local-semilocal convergence analysis and applications for two-point Newton-like methods in Banach space. J. Math. Anal. Appl. 2004, 298, 374-397. [CrossRef]

13. Argyros, I.K.; Cho, Y.J.; George, S. On the "Terra incognita" for the Newton-Kantrovich method. J. Korean Math. Soc. 2014, 51, 251-266. [CrossRef]

14. Argyros, I.K.; Cho, Y.J.; George, S. Local convergence for some third-order iterative methods under weak conditions. J. Korean Math. Soc. 2016, 53, 781-793. [CrossRef]

15. Ren, H.; Argyros, I.K.; Cho, Y.J. Semi-local convergence of Steffensen-type algorithms for solving nonlinear equations. Numer. Funct. Anal. Optim. 2014, 35, 1476-1499. [CrossRef]

16. Ezquerro, J.A.; Hernández, M.A.; Salanova, M.A. A discretization scheme for some conservative problems. J. Comput. Appl. Math. 2000, 115, 181-192. [CrossRef]

17. Ezquerro, J.A.; Hernández, M.A.; Salanova, M.A. A newton like method for solving some boundery value problems. Numer. Funct. Anal. Optim. 2002, 23, 791-805. [CrossRef]

18. Ezquerro, J.A.; Hernández, M.A. Generalized differentiability conditions for Newton's method. IMA J. Numer. Anal. 2002, 22, 187-205. [CrossRef]

19. Proinov, P.D. New general convergence theory for iterative process and its applications to NewtonKantorovich type theores. J. Complex. 2010, 26, 3-42. [CrossRef]

20. Sahu, D.R.; Yao, J.C.; Singh, V.K.; Kumar, S. Semilocal Convergence Analysis of S-iteration Process of Newton-Kantorovich Like in Banach Spaces. J. Optim. Theory Appl. 2017, 172, 102-127. [CrossRef] 
21. Parhi, S.K.; Gupta, D.K. Convergence of a third order method for fixed points in Banach spaces. Numer. Algorithms 2012, 60, 419-434. [CrossRef]

22. Agarwal, R.P.; O'Regan, D.; Sahu, D.R. Fixed Point Theory for Lipschitzian-Type Mappings with Applications; Topological Fixed Point Theory and its Applications; Springer: New York, NY, USA, 2009; p. 6.

23. Agarwal, R.P.; Meehan, M.; O'Regan, D. Fixed Point Theory and Applications; Cambridge University Press: Cambridge, UK, 2004.

24. Picard, E. Memorire sur la theorie des equations aux derivees partielles et la methode aes approximations successive. J. Math. Pures Appl. 1980, 6, 145-210.

25. Cho, Y.J. Survey on metric fixed point theory and applications. In Advances on Real and Complex Analysis with Applications; Trends in Mathematics; Ruzhahsky, M., Cho, Y.J., Agarwal, P., Area, I., Eds.; Birkhäuser, Springer: Basel, Switzerland, 2017.

26. Granas, A.; Dugundji, J. Fixed Point Theory; Springer: New York, NY, USA, 2003.

27. Zeidler, E. Nonlinear Functional Analysis and Its Applications I: Fixed-Point Theorems; Springer: New York, NY, USA, 1986.

28. Zeidler, E. Nonlinear Functional Analysis and Its Applications III: Variational Methods and Applications; Springer: New York, NY, USA, 1985.

29. Argyros, I.K. On Newton's method under mild differentiability conditions and applications. Appl. Math. Comput. 1999, 102, 177-183. [CrossRef]

30. Ortega, J.M.; Rheinboldt, W.C. Iterative Solution of Nonlinear Equations in Several Variables; Academic Press: New York, NY, USA; London, UK, 1970.

31. Bartle, R.G. Newton's method in Banach spaces. Proc. Am. Math. Soc. 1955, 6, 827-831.

32. Rall, L.B. Convergence of Stirling's method in Banaeh spaces. Aequa. Math. 1975, 12, 12-20. [CrossRef]

33. Parhi, S.K.; Gupta, D.K. Semilocal convergence of a Stirling-like method in Banach spaces. Int. J. Comput. Methods 2010, 7, 215-228. [CrossRef]

34. Argyros, I.K.; Muruster, S.; George, S. On the Convergence of Stirling's Method for Fixed Points Under Not Necessarily Contractive Hypotheses. Int. J. Appl. Comput. Math. 2017, 3, 1071-1081. [CrossRef]

35. Alshomrani, A.S.; Maroju, P.; Behl, R. Convergence of a Stirling-like method for fixed points in Banach spaces. J. Comput. Appl. Math. 2018. [CrossRef]

36. Parhi, S.K.; Gupta, D.K. A third order method for fixed points in Banach spaces. J. Math. Anal. Appl. 2009, 359, 642-652. [CrossRef]

37. Rall, L.B. Computational Solution of Nonlinear Operator Equations; John Wiley and Sons: New York, NY, USA, 1969.

38. Hernández, M.A.; Salanova, M.A. A Newton-like iterative process for the numerical solution of Fredholm nonlinear integral equations. J. Integr. Equ. Appl. 2005, 17, 1-17. [CrossRef]

39. Kohaupt, L. A Newton-like method for the numerical solution of nonlinear Fredholm-type operator equations. Appl. Math. Comput. 2012, 218, 10129-10148. [CrossRef] 